\title{
Coupling of multiple coulomb scattering with energy loss and straggling in HZETRN
}

\author{
Christopher J. Mertens ${ }^{\mathrm{a}}{ }^{,}$, John W. Wilson ${ }^{\mathrm{a}}$, Steven A. Walker ${ }^{\mathrm{b}}$, and John Tweed ${ }^{\mathrm{b}}$ \\ ${ }^{a}$ NASA Langley Research Center, Hampton, Virginia 23681-2199, United States \\ ${ }^{\mathrm{b}}$ Old Dominion University, Norfolk, Virginia 23529, United States
}

\begin{abstract}
The new version of the HZETRN deterministic transport code based on Green's function methods, and the incorporation of ground-based laboratory boundary conditions, has lead to the development of analytical and numerical procedures to include off-axis dispersion of primary ion beams due to small-angle multiple Coulomb scattering. In this paper we present the theoretical formulation and computational procedures to compute ion beam broadening and a methodology towards achieving a self-consistent approach to coupling multiple scattering interactions with ionization energy loss and straggling. Our initial benchmark case is a $60 \mathrm{MeV}$ proton beam on muscle tissue, for which we can compare various attributes of beam broadening with Monte Carlo simulations reported in the open literature.
\end{abstract}

\section{Introduction}

Current developments in the High Charge and Energy Transport (HZETRN) model are focused towards a full three-dimensional and computationally efficient deterministic transport code, capable of simulating HZE radiation transport with either space or ground-based laboratory boundary conditions (Wilson et al., 2005). Two parallel development paths of HZETRN have ensued. The first development path for HZETRN was based on marching procedures adequate for boundary conditions found in the space environment. For nearly two decades, this version of HZETRN has been validated in the space environment by comparing to a wide variety of spaceflight measurements taken on the Space Transportation System (STS) and the International Space Station (ISS). A complementary development path for HZETRN has been initiated in recent years based on a Green's function approach (Tweed et al., 2005) combined with nonperturbative techniques (Wilson et al., 1994). In addition to computation efficiency, an advantage of the Green's function approach is the ease and flexibility by which both ground-based laboratory and space environment boundary conditions can be incorporated into a single theoretical formulation. Ground-based laboratory boundary conditions are characterized by directed beams of ions of specific energy with detailed diagnostic particle spectrometer devices.

One aspect of the new version of HZETRN is the incorporation of off-axis dispersion of the primary ion beam for ground-based directed-beam applications. The beam broadening mechanism is attributed to small-angle, multiple Coulomb scattering of the incident ions by the target material nuclei. While the effects of multiple scattering are negligible in the space radiation environment, multiple scattering must be included in directed-beam 
applications and ground-based laboratory transport code simulations. To name a few applications, multiple scattering mechanisms must be included in directed-beam ion transport codes in order to accurately model and interpret the response of particle spectrometer devices in ion beam experiments, to simulate the physical and biologically important radiation dose, and to develop new methods and strategies for light ion beam radiation therapy.

In this paper we present the theoretical formalism and computation procedures for incorporating small-angle, multiple Coulomb scattering into the deterministic HZETRN code, and the method of coupling the ion-nuclear scattering interactions with ionization energy loss and straggling. Simulations of the effects of multiple scattering on ion beam broadening characterization will be computed for a $60 \mathrm{MeV}$ proton beam incident on muscle tissue. This example has important practical applications for improving knowledge in radiation biology and proton beam radiation therapy. Furthermore, this example will provide our initial benchmark to compare our new computation procedures with Monte Carlo simulations reported by Noshad and Givechi (2005). We calculate the effects of multiple scattering on path-length corrections, lateral beam broadening, and absorbed dose.

\section{The Boltzmann Transport Equation}

Beam broadening is attributed to the accumulation of many random, small-angle scattering events by which a particle from the incident ion beam is deflected by the average Coulomb potential established by the constituent nuclei of the target material and partially shielded by the orbital electrons. Thus, the propagation and broadening of an ion beam due to a random distribution of many scattering events, as described above, can be formulated in terms of the transport of a statistical distribution function. Specifically, transport of HZE ions is described by the linear Boltzmann equation, which can be derived on the basis of conservation principles (Wilson et al., 1991). Considering only ion-nucleus Coulomb scattering interactions, the transport of an ion beam is described by

$$
\grave{\mathbf{U}} \bullet \nabla \Phi(\mathbf{x}, \grave{\mathbf{U}}, E)=\int \sigma\left(\grave{\mathbf{U}}, \grave{\mathbf{U}}^{\prime}, \mathrm{E}, \mathrm{E}^{\prime}\right) \Phi\left(\mathbf{x}, \grave{\mathbf{U}}^{\prime}, E^{\prime}\right) d \grave{\mathbf{U}}^{\prime} d E^{\prime}-\sigma(E) \Phi(\mathbf{x}, \grave{\mathbf{U}}, E),
$$

where $\Phi(\mathbf{x}, \grave{\mathbf{U}}, E)$ is the flux of ions at position $\mathbf{x}$, propagating in direction $\boldsymbol{\Omega}$ with energy E. The differential and total macroscopic cross sections for ion-nucleus Coulomb scattering are denoted $\sigma\left(\grave{\mathbf{U}}_{,} \grave{\mathbf{U}}^{\prime}, \mathrm{E}, \mathrm{E}^{\prime}\right)$ and $\sigma(E)$, respectively. The first term on the righthand-side of (1) describes the production per volume of particles at position $\mathbf{x}$ propagating in direction $\mathbf{U}$ with energy $E$ due to Coulomb scattering of particles initially at position $\mathbf{x}$ propagating in direction $\dot{\mathbf{U}}^{\prime}$ with energy $E^{\prime}$. The second term on the right-hand-side of (1) describes of the loss of particles per volume due to scattering of

particles at position $\mathbf{x}$ in direction $\mathbf{U}$ with $E$ out of the beam. The net gain of particles per volume due to scattering, as described by the right-hand-side of (1), is identified with the infinitesimal change per unit length of particle flux at position $\mathbf{x}$ propagating in direction U with energy $E$, which is described by the left-hand-side of (1).

\section{Reduction to a Fokker-Planck Diffusion Equation}


In this section we invoke two approximations that simplify both the scattering cross section and the Boltzmann transport equation in (1). The first approximation is that the Coulomb scattering is elastic. Thus, the differential Coulomb scattering in (1) takes the form

$$
\sigma\left(\grave{\mathbf{U}}, \grave{\mathbf{U}}^{\prime}, E, E^{\prime}\right)=\sigma\left(\grave{\mathbf{U}}, \grave{\mathbf{U}}^{\prime}, E\right) \delta\left(E-E^{\prime}\right)
$$

The differential scattering cross section for elastic Coulomb scattering from a fixed target nuclei is the Rutherford differential scattering cross section (Rossi and Greisen, 1941). In the small-angle scattering limit, our second approximation, the Rutherford differential cross section depends only on the scattering angle - i.e., the angle between the incident particle direction and the direction of the scattered particle, and is given by

$$
\sigma\left(\grave{\mathbf{U}}, \grave{\mathbf{U}}^{\prime}, E\right)=\sigma\left(\grave{\mathbf{U}} \cdot \grave{\mathbf{U}}^{\prime}, E\right)=\sigma(\theta, E)=4 N_{A} \frac{\left(Z_{T} Z_{P}\right)^{2}}{A_{T}} \frac{\left(r_{o} \mu_{e}\right)^{2}}{(\mathrm{v} \cdot p)^{2}} \frac{1}{\theta^{4}}
$$

where $N_{A}$ is Avogadro's number; $A_{T}$ is the target molecular weight; $Z_{T}$ and $Z_{P}$ are the target and projectile atomic numbers, respectively; $r_{o}$ is the classical electron radius; $\mu_{\mathrm{e}}$ is the electron rest mass; $v$ and $p$ are the projectile velocity and momentum, respectively; and $\theta$ is the scattering angle. Note that the expression for the differential cross section implies that our unit of length is $\mathrm{g} / \mathrm{cm}^{2}$.

The elastic scattering approximation and the small-angle limit reduce the form of the differential scattering cross section to the expressions in (2) and (3), which simply the transport equation in (1). The small-angle limit will yield additional simplifications to the transport equation, as outlined below.

Consider the ion beam incident at the origin and propagating along the positive z-axis. The components of the unit vector oriented along the propagating direction, in terms of our coordinate system, are given by

$$
\grave{\mathbf{U}}=\sin \theta \cos \beta \hat{x}+\sin \theta \sin \beta \hat{y}+\cos \theta \hat{z}
$$

where $\theta$ is the angle between the z-axis and $\Omega$, and $\beta$ is the azimuth angle. It's useful to define orthogonal projections of the scattering angle $(\theta)$ on a plane perpendicular to the initial beam direction (i.e., the z-axis):

$$
\begin{aligned}
& \theta_{x} \equiv \sin \theta \cos \beta \\
& \theta_{y} \equiv \sin \theta \sin \beta .
\end{aligned}
$$

In the small-angle limit of $\theta \quad 1$, the projection angles are approximated by

$$
\theta_{x} \approx \theta \cos \beta
$$




$$
\theta_{y} \approx \theta \sin \beta
$$

such that

$$
\theta^{2} \approx \theta_{x}^{2}+\theta_{y}^{2}
$$

Moreover, the drift operator on the left-hand side of (1) reduces to

$$
\grave{\mathbf{U}} \bullet \nabla \approx \theta_{x} \frac{\partial}{\partial x}+\theta_{y} \frac{\partial}{\partial y}+\frac{\partial}{\partial z}
$$

Considering the approximations and definitions discussed above - i.e, the form of the differential elastic scattering cross section in (2) and (3) and the drift operator in (10) the transport equation in (1) can be written as

$$
\left[\frac{\partial}{\partial z}+\theta_{x} \frac{\partial}{\partial x}+\theta_{y} \frac{\partial}{\partial y}\right] \Phi\left(x, y, z ; \theta_{x}, \theta_{y}\right)=\int_{0}^{2 \pi} d \beta \int_{0}^{\pi} \sigma(\theta) \theta d \theta\left[\Phi\left(x, y, z ; \theta_{x}^{\prime}, \theta_{y}^{\prime}\right)-\Phi\left(x, y, z ; \theta_{x}, \theta_{y}\right)\right]
$$

A diffusion equation can be derived by making the usual Fokker-Planck approximation by expanding the expression in brackets, (11), in a Taylor series and retaining no terms beyond the second derivatives (Scott, 1949). The Fokker-Planck approximation is justified in the small-angle limit. Realizing that $\left(\theta_{x}^{\prime}, \theta_{y}^{\prime}\right)$ represent the projected angles before scattering into $\left(\theta_{x}, \theta_{y}\right)$ we have

$$
\begin{aligned}
& \theta_{x}^{\prime}=\theta_{x}-\theta \cos \beta \\
& \theta_{y}^{\prime}=\theta_{y}-\theta \sin \beta
\end{aligned}
$$

Thus, the Taylor series expansion of the ion flux distribution is written

$$
\begin{aligned}
\Phi\left(x, y, z ; \theta_{x}^{\prime}, \theta_{y}^{\prime}\right)= & \Phi\left(x, y, z ; \theta_{x}, \theta_{y}\right)-\theta \cos \beta \frac{\partial \Phi}{\partial \theta_{x}}-\theta \sin \beta \frac{\partial \Phi}{\partial \theta_{y}} \\
& +\frac{\theta^{2} \cos ^{2} \beta}{2} \frac{\partial^{2} \Phi}{\partial \theta_{x}^{2}}+\frac{\theta^{2} \sin ^{2} \beta}{2} \frac{\partial^{2} \Phi}{\partial \theta_{y}{ }^{2}}+\cdots
\end{aligned}
$$

Substitute (14) into the right hand side of (11) and integrate over solid angle $\left(d \Omega^{\prime} \approx \theta d \theta d \beta\right)$. The result is

$$
\left[\frac{\partial}{\partial z}+\theta_{x} \frac{\partial}{\partial x}+\theta_{y} \frac{\partial}{\partial y}\right] \Phi\left(x, y, z ; \theta_{x}, \theta_{y}\right)=\frac{1}{\lambda_{s}(z)}\left[\frac{\partial^{2}}{\partial \theta_{x}^{2}}+\frac{\partial^{2}}{\partial \theta_{y}^{2}}\right] \Phi\left(x, y, z ; \theta_{x}, \theta_{y}\right)
$$


where

$$
\begin{aligned}
\frac{1}{\lambda_{S}(z)} & =\frac{1}{4} \int_{0}^{\pi} \sigma(\theta(z)) \theta^{3} d \theta=\frac{1}{4} \frac{d\left\langle\theta^{2}(z)\right\rangle}{d z} \\
& =16 \pi N_{A} \frac{\left(Z_{T} Z_{P}\right)^{2}}{A_{T}} \frac{\left(r_{o} \mu_{e}\right)^{2}}{(\mathrm{v}(z) p(z))^{2}} \ln \left(181 Z_{T}^{-1 / 3}\right) .
\end{aligned}
$$

The characteristic scattering length is given by $\lambda_{s}$, which is equal to the inverse of the diffusion coefficient, and is related to the mean-square scattering angle per unit thickness (dz), as (16) indicates. Equation (16) can be readily extended to include multi-component target materials, such as muscle tissue discussed below, by the expression

$$
\frac{1}{\lambda_{s}(z)}=16 \pi Z_{P}^{2} \frac{\left(r_{o} \mu_{e}\right)^{2}}{(\mathrm{v}(z) p(z))^{2}} \sum_{m} \rho_{A}(m) Z_{T}^{2}(m) \ln \left(181 Z_{T}^{-1 / 3}(m)\right)
$$

where the sum is over the chemical constituents of the target material and $\rho_{\mathrm{A}}$ is the number of atoms per gram.

We have tacitly coupled energy loss in the Fokker-Planck partial differential equation in (15) by letting the diffusion coefficient depend on the propagation depth (z), and by letting the scattering angle depend on propagation depth in (16). The expression for the characteristic scattering length in (16) was evaluated using the approximations employed by Rossi and Greisen (1941): the lower limit of integration in (16) was set by considering the screening of the nuclear charge by the orbital electrons using the Thomas-Fermi statistical model of the average Coulomb potential function; and the upper limit of integration in (16) was set by considering the finite nuclear size and assuming a uniform spherically symmetric nuclear charge distribution.

The energy loss of a projectile particle is due to energy transfer to the orbital electrons of the target material. Using the continuous slowing down approximation, the mean energy of the ion beam after propagating a distance $\mathrm{z}$ into the target material is given by the usual range-energy relation (Wilson et al., 1991)

$$
\langle E(z)\rangle=R^{-1}\left[R\left(E_{0}\right)-z\right]
$$

where $\mathrm{R}(\mathrm{Eo})$ is the mean range of an incident particle at the initial energy Eo at zero depth, and the unit of energy is taken to be MeV/nucleon. The velocity and momentum at each propagation depth-z in (16) can be evaluated using the mean beam energy from (18) and the relativistic energy relations, such that

$$
\beta(z) \equiv \mathrm{v}(z) / c=\left[1-\left(1+\langle E(z)\rangle / \mathrm{amu} \cdot \mathrm{c}^{2}\right)^{-2}\right]^{1 / 2}
$$




$$
p(z) c=A_{p} \cdot\left(\mathrm{amu} \cdot \mathrm{c}^{2}\right) \cdot \beta(z) \cdot\left(1-\beta^{2}(z)\right)^{-1 / 2} .
$$

In the above equations, $c$ is the speed of light, $A_{p}$ is the molecular weight of the projectile particle, and $a m u=931.5 \mathrm{Mev} / \mathrm{c}^{2}$ (atomic mass unit).

\section{Solution of Depth-Dependent Fokker-Planck Equation}

The 3-D Fokker-Planck equation in (15) can be separated into two independent partial differential equations in the two sets of projected coordinates $-x, \theta \mathrm{x}$ and $\mathrm{y}, \theta \mathrm{y}-\mathrm{by}$ factoring the flux distribution into a product of projected flux distributions, i.e.,

$$
\Phi\left(x, y, z ; \theta_{x}, \theta_{y}\right)=\Phi\left(z, x ; \theta_{x}\right) \Phi\left(z, y ; \theta_{y}\right) .
$$

For the simple boundary conditions of an infinitesimal pencil beam of unit flux at the origin, i.e.,

$$
\begin{gathered}
\Phi\left(0, x, \theta_{x}\right)=\delta(x) \delta\left(\theta_{x}\right) \\
\Phi\left(0, y, \theta_{y}\right)=\delta(y) \delta\left(\theta_{y}\right),
\end{gathered}
$$

Eyges (1948) found the solutions of the depth-dependent Fokker-Planck partial differential equations, which are referred to as the Fermi-Eyges distribution functions. Thus, the solutions for the projected flux distributions are

$$
\begin{aligned}
& \Phi\left(z, x, \theta_{x}\right)=\frac{1}{4 \pi \sqrt{B(z)}} \exp \left[\frac{-\left(A_{0}(z) x^{2}-2 x \theta_{x} A_{1}(z)+A_{2}(z) \theta_{x}^{2}\right)}{4 B(z)}\right] \\
& \Phi\left(z, y, \theta_{y}\right)=\frac{1}{4 \pi \sqrt{B(z)}} \exp \left[\frac{-\left(A_{0}(z) y^{2}-2 y \theta_{y} A_{1}(z)+A_{2}(z) \theta_{y}^{2}\right)}{4 B(z)}\right]
\end{aligned}
$$

where

$$
B(z)=A_{0}(z) A_{2}(z)-A_{1}^{2}(z)
$$

The projected-flux distributions in (24) and (25) resemble 2-variate Gaussian probability distributions in angular and lateral displacements, with a non-zero correlation between the angular and lateral displacements. The similarity of the projected-flux distributions with 2-variate Gaussian probability distributions is consistent with our physical concept and formulation of a localized initial ion beam that is broadened with propagation distance in its angular width and lateral displacements due to a continuous distribution of random, small-angle scattering events. This analogy is further strengthened by the fact that the A-functions in (24) and (25) are integral moments of the diffusion coefficient, which can be expressed in terms of the usual statistical moments. Specifically, 


$$
\begin{gathered}
2 A_{0}(z)=\left\langle\theta_{y}^{2}(z)\right\rangle=2 \int_{0}^{z} \frac{d z^{\prime}}{\lambda_{S}\left(z^{\prime}\right)}=\frac{1}{2} \int_{0}^{z} \frac{d\left\langle\theta^{2}\left(z^{\prime}\right)\right\rangle}{d z^{\prime}} d z^{\prime} \\
2 A_{1}(z)=\left\langle y \theta_{y}(z)\right\rangle=2 \int_{0}^{z} \frac{\left(z-z^{\prime}\right) d z^{\prime}}{\lambda_{S}\left(z^{\prime}\right)}=\frac{1}{2} \int_{0}^{z}\left(z-z^{\prime}\right) \frac{d\left\langle\theta^{2}\left(z^{\prime}\right)\right\rangle}{d z^{\prime}} d z^{\prime} \\
2 A_{2}(z)=\left\langle y^{2}(z)\right\rangle=2 \int_{0}^{z} \frac{\left(z-z^{\prime}\right)^{2} d z^{\prime}}{\lambda_{S}\left(z^{\prime}\right)}=\frac{1}{2} \int_{0}^{z}\left(z-z^{\prime}\right)^{2} \frac{d\left\langle\theta^{2}\left(z^{\prime}\right)\right\rangle}{d z^{\prime}} d z^{\prime} .
\end{gathered}
$$

Similar equations hold for the statistical moments projected on the $\mathrm{z}-\mathrm{x}$ and $\mathrm{z}-\theta \mathrm{x}$ planes.

To accommodate realistic boundary conditions, and in order to calculate the path-length corrections in section 5, more general solutions for the Fermi-Eyges distributions are required. We extend the boundary condition in (22) and (23) to describe an infinitesimal pencil beam of unit flux with both non-zero angular displacements $\left(\theta \mathrm{x}_{\mathrm{o}}\right.$ and $\left.\theta \mathrm{y}_{\mathrm{o}}\right)$ and nonzero lateral displacements $\left(x_{o}\right.$ and $\left.y_{o}\right)$. That is, we take

$$
\begin{gathered}
\Phi\left(0, x, \theta_{x}\right)=\delta\left(x-x_{0}\right) \delta\left(\theta_{x}-\theta_{x_{0}}\right) \\
\Phi\left(0, y, \theta_{y}\right)=\delta\left(y-y_{0}\right) \delta\left(\theta_{y}-\theta_{y_{0}}\right)
\end{gathered}
$$

Generalized Fermi-Eyges projected-flux distribution functions can be obtained by solving the projected Fokker-Planck partial differential equations subject to the boundary conditions expressed in (30) and (31). The form of the generalized Fermi-Eyges distribution functions are the same as (24) and (25) provided the following variable replacements are made:

$$
\begin{gathered}
x \rightarrow\left(x-x_{0}-\theta_{x_{0}} z\right) ; \theta_{x} \rightarrow\left(\theta_{x}-\theta_{x_{0}}\right) \\
y \rightarrow\left(y-y_{0}-\theta_{y_{0}} z\right) ; \theta_{y} \rightarrow\left(\theta_{y}-\theta_{y_{0}}\right) .
\end{gathered}
$$

The statistical moments in (27)-(29) can also be computed from the generalize FermiEyges distribution functions. Statistical boundary conditions can be accommodated by replacing the initial angular and lateral displacements by an ensemble average such that (Hollmark et al., 2004)

$$
\begin{gathered}
\left\langle\theta^{2}(z)\right\rangle=\left\langle\theta^{2}(0)\right\rangle+\int_{0}^{z} \frac{d\left\langle\theta^{2}\left(z^{\prime}\right)\right\rangle}{d z^{\prime}} d z^{\prime} \\
\left\langle r^{2}(z)\right\rangle=\left\langle r^{2}(0)\right\rangle+2\langle r \theta(0)\rangle z+\left\langle\theta^{2}(0)\right\rangle z^{2}+\int_{0}^{z}\left(z-z^{\prime}\right)^{2} \frac{d\left\langle\theta^{2}\left(z^{\prime}\right)\right\rangle}{d z^{\prime}} d z^{\prime} \\
\langle r \theta(z)\rangle=\langle r \theta(0)\rangle+\left\langle\theta^{2}(0)\right\rangle z+\int_{0}^{z}\left(z-z^{\prime}\right) \frac{d\left\langle\theta^{2}\left(z^{\prime}\right)\right\rangle}{d z^{\prime}} d z^{\prime} .
\end{gathered}
$$

The above equations, (34)-(36), are transport integrals that describe beam broadening characteristics for arbitrary boundary conditions. We have assumed, for simplicity, cylindrical symmetry in deriving (34)-(36). Asymmetries can be accounted for by 
developing the transport integrals for the statistical moments in the projected $\mathrm{z}-\mathrm{x} / \mathrm{x} \mathrm{x}$ and $\mathrm{z}-\mathrm{y} / \theta \mathrm{y}$ planes separately. For this study, however, we will assume cylindrical symmetry.

In Figure 1 and Figure 2 we show, respectively, the radial broadening and the radial flux distribution for an initial infinitesimal $60 \mathrm{MeV}$ proton beam incident on muscle tissue.

The longitudinal propagation extends to $31 \mathrm{~mm}$. The Bragg peak is located at $30.49 \mathrm{~mm}$ (shown in Figure 8). The chemical composition of muscle tissue was considered as $\mathrm{H}(63 \%)+\mathrm{C}(6 \%)+\mathrm{N}(1 \%)+\mathrm{O}(28 \%)$, and its density was considered to be equal to $1 \mathrm{~g} / \mathrm{cm}^{3}$ (Noshad and Givechi, 2005). The radial flux distribution is obtained from the projected Fermi-Eyges distributions functions by

$$
\begin{aligned}
f(x, y, z) & =\int_{-\infty}^{+\infty} d \theta_{x} \int_{-\infty}^{+\infty} d \theta_{y} \Phi\left(z, x, \theta_{x}\right) \Phi\left(z, y, \theta_{y}\right) \\
& =\frac{1}{\pi\left\langle r^{2}(z)\right\rangle} \exp \left(\frac{-\left(x^{2}+y^{2}\right)}{\left\langle r^{2}(z)\right\rangle}\right) .
\end{aligned}
$$

Results for the $60 \mathrm{MeV}$ proton beam on muscle tissue described above were computed by the TRIM Monte Carlo code and reported by Noshad and Givechi (2005). The radial broadening at the Bragg peak in Figure 1 is $2.13 \mathrm{~mm}$, which is in excellent agreement with the results calculated by Noshad and Givechi (hereafter referred to as NG). However, the broadening at intermediate tissue depths and the rate of broadening is substantially different between NG and our calculations. Our radial broadening is greater than the NG results for tissue depths less than the Bragg Peak. These differences are relatively small below $20 \mathrm{~mm}$ tissue depth, but appear to be on the order of $50 \%$ at around $25 \mathrm{~mm}$. The radial broadening computed by NG rapidly increases between $25 \mathrm{~mm}$ and the Bragg Peak.

Figure 2 shows the radial flux distribution for an initial infinitesimal $60 \mathrm{MeV}$ proton beam on muscle tissue at various tissue depths. The flux is shown along one of the lateral axis (either $\mathrm{x}$ - or $\mathrm{y}$-axis). The proton beam is highly localized at $5 \mathrm{~mm}$ depth where the radial broadening is only $0.12 \mathrm{~mm}$. At $15 \mathrm{~mm}, 25 \mathrm{~mm}$, and $30 \mathrm{~mm}$ tissue depths, the radial broadening is $0.64 \mathrm{~mm}, 1.48 \mathrm{~mm}$, and $2.06 \mathrm{~mm}$, respectively.

In Figure 3 and Figure 4 we show, respectively, the radial broadening and the radial flux distribution for an initial finite width $60 \mathrm{MeV}$ proton beam on muscle tissue. The initial beam width is $2 \mathrm{~mm}$ in diameter. In this case our radial broadening results are less than those computed by NG. The largest differences occur for tissue depths greater than 20 $\mathrm{mm}$. We find a radial broadening of $2.35 \mathrm{~mm}$ at the Bragg Peak, while the radial broadening is closer to $3.00 \mathrm{~mm}$ for the result reported by NG. Similar to the initial infinitesimal beam case, the NG radial broadening increases at a slow rate at small to intermediate tissue depths, but increases rapidly near the end-of-range, particularly between $20 \mathrm{~mm}$ and the Bragg peak for the initial $2 \mathrm{~mm}$ diameter proton beam.

Figure 4 shows the radial flux distribution for an initial $2 \mathrm{~mm}$ diameter $60 \mathrm{MeV}$ proton beam on muscle tissue at various tissue depths. The flux is shown along one of the lateral 
axis (either $\mathrm{x}$ - or $\mathrm{y}$-axis). The radial broadening at $5 \mathrm{~mm}, 15 \mathrm{~mm}, 25 \mathrm{~mm}$, and $30 \mathrm{~mm}$ tissue depths is $1.01 \mathrm{~mm}, 1.19 \mathrm{~mm}, 1.79 \mathrm{~mm}$, and $2.29 \mathrm{~mm}$, respectively.

\section{Path-Length Correction}

To summarize the theoretical formulation of sections 3 and 4, energy loss was coupled to beam broadening by solving the multiple scattering transport equation in (15) with the depth-dependent diffusion coefficient given by (17), using (19)-(20). The diffusion coefficient is a function of depth due to ionization energy loss and is calculated assuming the continuous slowing down approximation in (18). However, the actual particle path in the target material is greater than the physical depth-z in the material due to multiple scattering processes. A necessary step towards a self-consistent theory of multiple scattering and energy loss is to compute a path-length correction to the longitudinal depth in the evaluation of the mean energy of the beam particles, prior to computing the transport integrals in (34)-(36) and the flux distributions in (24)-(25), and (37).

Bichsel and Uehling (1960) defined an effective 1-D path-length correction which is a function of the projected mean-square scattering angle,

$$
\langle\Delta R(z)\rangle=\int_{0}^{z}\left\langle\theta_{x}^{2}\left(z^{\prime}\right)\right\rangle d z^{\prime}=\int_{0}^{z}\left\langle\theta_{y}^{2}\left(z^{\prime}\right)\right\rangle d z^{\prime}
$$

The projected mean-square scattering angle at intermediate depths- $z^{\prime}$ depends on the boundary conditions at zero depth and at depth-z, which can be formulated in terms of a doubly-conditional probability distribution

$$
\left\langle\theta_{y}^{2}\left(z^{\prime}\right)\right\rangle=\int_{-\infty}^{+\infty} P\left(\theta_{y}^{\prime} ; z^{\prime} \mid 0, y_{0}, \theta_{0} ; z, y_{1}, \theta_{1}\right) \theta_{y}^{\prime 2} d \theta_{y}^{\prime}
$$

The probability function in (39) describes the probability that at intermediate depth- $z^{\prime}$ a beam particle is propagating with an angular divergence of $\theta_{y}^{\prime}$, given that the particle had lateral displacement $\mathrm{y}_{\mathrm{o}}$ and angular displacement $\theta_{\mathrm{o}}$ at zero depth $(\mathrm{z}=0)$, and emerges at depth-z with a lateral displacement $\mathrm{y}_{1}$ and an angular displacement $\theta_{1}$.

The influence of initial beam statistics has already been included in the development of the transport integrals in (34)-(36). Therefore, without loss of generality, we can set $y_{o}$ and $\theta_{\mathrm{o}}$ equal to zero in (39). The emerging boundary conditions at depth-z require a little more insight. Recall that the goal is to develop an effective 1-D propagation depth in the target material. Thus, we develop a correction to the physical depth-z - i.e., the effective 1-D propagation depth - by computing a path-length correction due to multiple scattering of particles that emerge at depth-z with zero angular displacement, irrespective of the lateral displacement. Consequently, for the boundary conditions just described, the doubly-conditional probability distribution in (39) becomes 


$$
\begin{aligned}
P\left(\theta_{y}^{\prime}\left(z^{\prime}\right)\right) d \theta_{y}^{\prime} & \equiv \int_{-\infty}^{+\infty} d y_{1} P\left(\theta_{y}^{\prime} ; z^{\prime} \mid 0,0,0 ; z, y_{1}, 0\right) \\
& =\frac{d \theta_{y}^{\prime} \int_{-\infty}^{+\infty} d y_{1} \int_{-\infty}^{+\infty} d y^{\prime} \Phi\left(z-z^{\prime}, y_{1}-y^{\prime}-\theta_{y}^{\prime}\left(z-z^{\prime}\right),-\theta_{y}^{\prime}\right) \Phi\left(z^{\prime}, y^{\prime}, \theta_{y}^{\prime}\right)}{\int_{-\infty}^{+\infty} d y_{1} \Phi\left(z, y_{1}, 0\right)} \\
& =d \theta_{y}^{\prime}\left(\frac{A_{0}(z)}{4 \pi A_{0}\left(z-z^{\prime}\right) A_{0}\left(z^{\prime}\right)}\right)^{\frac{1}{2}} \exp \left(\frac{-\theta_{y}^{\prime 2}}{4}\left[\frac{1}{A_{0}\left(z-z^{\prime}\right)}+\frac{1}{A_{0}\left(z^{\prime}\right)}\right]\right) .
\end{aligned}
$$

Substitute (40) into (39), and (39) in to (38), such that

$$
\left\langle\theta_{y}^{\prime 2}\left(z^{\prime}\right)\right\rangle=\frac{2 A_{0}\left(z^{\prime}\right) A_{0}\left(z-z^{\prime}\right)}{A_{0}(z)}
$$

and

$$
\langle\Delta R(z)\rangle=\frac{2}{A_{0}(z)} \int_{0}^{z} A_{0}\left(z-z^{\prime}\right) A_{0}\left(z^{\prime}\right) d z^{\prime} .
$$

To get a rough idea of how the path-length correction in (42) depends on depth and the characteristic scattering length in (17), assume the scattering length is independent of depth. The statistical moments in (27)-(29) can be evaluated analytically, and (42) reduces to

$$
\langle\Delta R(z)\rangle \frac{z^{2}}{3 \lambda_{s}} .
$$

Figure 5 shows the percent increase in the penetration depth due to small-angle multiple scattering. At the end-of-range, the effective 1-D path-length is $0.8 \%$ greater than the physical depth-z. This may appear as a small correction, but it has a significant effect on the mean energy, which is decreasing non-linearly near the end-of range. The influence of multiple scattering on the mean energy is characterized by replacing (18) with the expression

$$
\langle E(z)\rangle=R^{-1}\left[R\left(E_{0}\right)-(z+\langle\Delta R(z)\rangle)\right] .
$$

Figure 6 shows the influence of the path-length correction on the mean energy. Since the effective 1-D propagation depth is greater than the physical depth-z, the mean energy due to multiple scattering processes is less than the mean energy without scattering.

Furthermore, the ion beam will reach the end-of-range at a smaller physical depth when the effects of multiple scattering on the propagation depths are included. For example, the minimum mean energy at the location of the Bragg peak when scattering effects are included is $0.14 \mathrm{MeV} /$ nucleon, and the Bragg peak is located at $30.49 \mathrm{~mm}$ tissue depth. If 
scattering effects are ignored, the mean energy at $30.49 \mathrm{~mm}$ is $3.95 \mathrm{MeV} /$ nucleon. This corresponds to nearly a factor of 30 overestimation of the mean energy due to the meager $0.8 \%$ increase in effective 1-D path-length due to multiple scattering processes, as described above. However, the overestimation in the Bragg peak location, if multiple scattering effects on path-length are ignored, is comparable to the error in effective 1-D path-length. Thus, the Bragg peak is located at $30.73 \mathrm{~mm}$ tissue depth if multiple scattering is ignored, corresponding to an overestimation of roughly $0.8 \%$

Energy straggling is also influenced by multiple scattering, through the effective 1-D path-length correction, since straggling is a function of the mean energy (Wilson et al. 2000, 2002). Figure 7 shows the increases in energy straggling as a result of multiple scattering path-length corrections for the $60 \mathrm{MeV}$ proton beam on muscle tissue. The energy straggling width increases with decreasing mean energy (Wilson et al., 2000, 2002). A smaller mean energy when multiple scattering effects are includes produces a larger straggling width. At the Bragg peak when multiple scattering processes are included (i.e., at $30.49 \mathrm{~mm}$ tissue depth), the straggling width is $2.21 \mathrm{MeV}$. If multiple scattering effects are not included, the straggling width is $2.10 \mathrm{MeV}$ at $30.49 \mathrm{~mm}$ tissue depth.

\section{Absorbed Dose Distribution}

In this section we describe the formulation to compute absorbed dose, which includes the coupling of multiple scattering with ionization energy loss and straggling.

\subsection{On-Axis Dose Distribution}

The on-axis dose distribution is given by the following expression

$$
d(z)=K \int_{0}^{\infty} S(E) \Phi(z, E) d E
$$

where $S(E)$ is the stopping power, $\Phi(\mathrm{z}, \mathrm{E})$ is the on-axis spectral flux distribution, and $K$ is a unit conversion factor (Wilson et al., 2005). The spectral flux distribution is obtained by a second-order moment expansion of the Boltzmann transport equation, which can be expressed in terms of a Gaussian function

$$
\Phi(z, E)=\frac{1}{\sqrt{2 \pi} s(z)} \exp \left[-\frac{(E-\langle E(z)\rangle)^{2}}{2 s^{2}(z)}\right]
$$

where the mean energy in (46) is evaluated using (44) and the energy straggling width $\mathrm{s}(\mathrm{z})$ is evaluated according to the global Payne formulation described by Wilson et al. (2000, 2002). Both the mean energy and straggling width include multiple scattering effects via the path-length correction, as describe in the previous section. An expression for the absorbed dose can be obtained by substituting (46) into (45): 


$$
d(z)=\frac{K}{2 \sqrt{\pi}} \sum_{m=0}^{q_{h}} H_{m} S\left[\langle E(z)\rangle+\sqrt{2} s(z) x_{m}^{h}\right]
$$

for penetration depths less than the particle range, and

$$
\begin{aligned}
d(R+\Delta z)= & \frac{K}{2 \sqrt{\pi}}\left\{\frac{\langle E(z)\rangle}{\sqrt{2} s(z)} \sum_{m=0}^{q_{g}} G_{m} \exp \left[-\frac{\langle E(z)\rangle^{2}}{8 s^{2}(z)}\left(1+x_{m}^{g}\right)^{2}\right] S\left[\frac{\langle E(z)\rangle}{2}\left(1-x_{m}^{g}\right)\right]\right\} \\
& +\frac{K}{2 \sqrt{\pi}} \sum_{m=0}^{q_{h}} H_{m} S\left[\langle E(z)\rangle+\sqrt{2} s(z) x_{m}^{h}\right]
\end{aligned}
$$

for penetration depths greater than the end-of-range. The above expressions assume incident unit particle flux. In (48) $\mathrm{R}$ is the actual mean range of the beam, including the effects of multiple scattering, and $\Delta z$ is the increment in depth beyond the mean range. In both (47) and (48), $\mathrm{H}_{\mathrm{m}}$ and $x_{m}^{h}$ are the $\mathrm{m}^{\text {th }}$ Gauss-Hermite quadrature weight and abscissa, respectively. In (48), $\mathrm{G}_{\mathrm{m}}$ and $x_{m}^{g}$ are the $\mathrm{m}^{\text {th }}$ Gauss-Legendre quadtrature weight and abscissa, respectively. The terms in (48) that involve the Gauss-Legendre weights and abscissas account for the statistical distribution of particles with energies greater than zero when the mean energy reaches zero at the end-of-range. The number of GaussHermite and Gauss-Legendre quadtrature points are denoted $\mathrm{q}_{\mathrm{h}}$ and $\mathrm{q}_{\mathrm{g}}$, respectively. Note: one can show that the orthogonal polynomials over the range $[0, \infty]$ are the standard Gauss-Hermite polynomials with the numerical quadrature formula specified by evaluating the standard Gauss-Hermite abscissas at only the positive values and to divide the standard quadrature weights by two.

Figure 8 shows the relative absorbed dose for the $60 \mathrm{MeV}$ proton beam on muscle tissue. The dose-depth curve in Figure 8 was calculated from (47) and (48) using a 5-point Gauss-Hermite quadrature rule and a 10-point Gauss-Legendre quadrature rule. The result is in excellent agreement with the Monte Carlo TRIM code calculation reported by NG. In section 5 it was shown by the minimum mean energy that the location of the Bragg peak is at a tissue depth of $30.49 \mathrm{~mm}$, which is consistent with the maximum in absorbed dose in Figure 8. NG report the Bragg peak at $30.5 \mathrm{~mm}$. Recall from section 5 that the Bragg peak would be located at $30.73 \mathrm{~mm}$ tissue depth if multiple scattering effects via path-length corrections was not included in the calculations.

\subsection{Off-Axis Dose Distribution}

The conceptual picture of ion beam transport is that the beam is composed of a collection of independent pencil beams propagating and broadening through the target material. Thus, the dose distribution is a summation of contributions from each pencil beam. This concept of the off-axis dose distribution can be formulated as follows (Hogstrom et al., 1981):

$$
D(x, y, z)=\iint S\left(x^{\prime}, y^{\prime}, z\right) d\left(x-x^{\prime}, y-y^{\prime}, z\right) d x^{\prime} d y^{\prime} .
$$


In the above equation, $S\left(x^{\prime}, y^{\prime}\right)$ is the relative strength of a pencil beam at $x^{\prime}$ and $y^{\prime}$. The contribution of dose at $x$ and $y$ from the pencil beam at $x^{\prime}$ and $y^{\prime}$ is specified by the dose function $d\left(x-x^{\prime}, y-y^{\prime}, z\right)$. The integration limits in (49) are specified by the 2-D lateral extent of the beam projected at depth-z, which includes the initial beam width and broadening due to multiple scattering.

The dose function in (49) is factored into an on-axis term, given by (45), which is modulated by an off-axis beam broadening function, given in terms of the radial FermiEyges distribution function, such that

$$
d(x, y, z)=f(x, y, z) d(z)
$$

where

$$
\begin{aligned}
f(x, y, z) & =\int_{-\infty}^{+\infty} d \theta_{x} \int_{-\infty}^{+\infty} d \theta_{y} \Phi\left(z, x, \theta_{x}\right) \Phi\left(z, y, \theta_{y}\right) \\
& =\frac{1}{\pi \sigma_{r}^{2}(z)} \exp \left(\frac{-\left(x^{2}+y^{2}\right)}{\sigma_{r}^{2}(z)}\right)
\end{aligned}
$$

and

$$
\sigma_{r}^{2}(z)=\int_{0}^{z}\left(z-z^{\prime}\right)^{2} \frac{d\left\langle\theta^{2}\left(z^{\prime}\right)\right\rangle}{d z^{\prime}} d z^{\prime}
$$

Notice that the multiple scattering distribution function in (51) and the transport integral in (52) have slightly different forms compared to the previous expressions in (37) and (35). In computing the off-axis dose distribution as formulated in (49), the influence of the finite initial boundary conditions are included in the limits of integration rather than in the transport integrals. Thus, the beam broadening function and the transport integral in (51) and (52) need only include broadening due to multiple scattering as a result of transport through the target material. The boundary condition on the double integral in (49) is the projected lateral extent of the broadened beam at depth-z, as previously mentioned.

As an example of an application of the above formulation, consider an initial finite beam of uniform unit flux distribution incident on the target material at $\mathrm{z}=0$. The relative strength of a pencil beam is specified by the following equation

$$
S(x, y, z)=\theta\left(x+\frac{d}{2}+\sqrt{\sigma_{r}^{2}(z)}\right) \theta\left(-x+\frac{d}{2}+\sqrt{\sigma_{r}^{2}(z)}\right) \theta\left(y+\frac{d}{2}+\sqrt{\sigma_{r}^{2}(z)}\right) \theta\left(-y+\frac{d}{2}+\sqrt{\sigma_{r}^{2}(z)}\right)
$$

In the above equation, $d$ is the diameter of the initial beam, and $\theta$ in this case denotes the Heavyside step function. Substitute (53), (50), and (51) into (49) to obtain the following result 


$$
\begin{aligned}
D(x, y, z) & =\frac{1}{4}\left(\operatorname{erf}\left[\frac{\sqrt{\left\langle r^{2}(z)\right\rangle}+x}{\sigma_{r}(z)}\right]+\operatorname{erf}\left[\frac{\sqrt{\left\langle r^{2}(z)\right\rangle}-x}{\sigma_{r}(z)}\right]\right) \\
& \mathrm{x}\left(\operatorname{erf}\left[\frac{\sqrt{\left\langle r^{2}(z)\right\rangle}+y}{\sigma_{r}(z)}\right]+\operatorname{erf}\left[\frac{\sqrt{\left\langle r^{2}(z)\right\rangle}-y}{\sigma_{r}(z)}\right]\right) d(z),
\end{aligned}
$$

where $\operatorname{erf}(x)$ is the error function, and the transport integrals are defined in (35) and (52), and the on-axis dose distribution is given by (47) and (48).

Figure 9 shows the relative lateral dose distribution at the Bragg peak (30.49 mm tissue depth) for a $60 \mathrm{MeV}$ proton beam on muscle tissue. The initial beam width was taken to be $10 \mathrm{~mm}$ and the dose distribution was computed using (54). This result is in reasonable agreement was the results presented by NG, which show the lateral distribution in terms of the number of protons. Our results are broader in the wings of the lateral distribution. The contribution to absorbed dose in the lateral direction in Figure 9 approaches zero at approximately $+/-9 \mathrm{~mm}$. The distribution shown by NG approach zero in the lateral distribution at a lateral displacement of about $+/-7 \mathrm{~mm}$. This result is to be expected since our radial broadening transport integral (i.e., (35)) tends to be larger than the TRIM results in the region between intermediate depths and the end-of-range, as discussed in section 4.

Figure 10 shows the lateral distribution of relative isodose contours for the $60 \mathrm{MeV}$ proton beam on muscle tissue. Broadening due to multiple scattering beyond the initial beam width is clearly evident at $26 \mathrm{~mm}$ tissue depth. As the end-of-range is approached, the beam rapidly broadens as the absorbed dose rapidly increases, and there is a welldefined cutoff in absorbed dose beyond the Bragg peak.

\section{Summary}

We have presented a 3-D formulation of ion beam multiple Coulomb scattering based on deterministic transport methods. The formulation includes an important step towards a self-consistent approach to coupling multiple scattering with ionization energy loss and straggling. Energy loss is coupled to lateral beam broadening due to multiple scattering by solving the Fokker-Planck transport equation with a depth-dependent, or equivalently, with an energy-dependent diffusion coefficient. The depth-dependent diffusion coefficient is evaluated by using the well-know range-energy relation. The range-energy relations itself is affected by multiple scattering processes as the actual particle pathlength is greater then the penetration depth, which is accounted for in our formulation using the path-length correction to calculate an effective 1-D penetration depth. The methodologies and computation procedures presented in this paper will soon be integrated into the new 3-D version of HZETRN, which accommodates both space environment and ground-based laboratory boundary conditions. 
We have tested our approach to ion beam broadening by calculating various broadening characteristics for a $60 \mathrm{MeV}$ proton beam on muscle tissue. This scenario has important applications in radiation biology and ion beam therapy. Furthermore, Noshad and

Givechi (2005) have calculated various broadening characteristics for the $60 \mathrm{MeV}$ proton beam on muscle tissue using the TRIM Monte Carlo code from which we can compare. The results between our deterministic code and the TRIM simulations generally compare quite favorably for the $60 \mathrm{MeV}$ proton beam on muscle tissue. Future efforts will be to extend our formulation to include inhomogeneous target materials and to incorporate beam attenuation due to nuclear absorption process, as well as a thorough transport code verification and validation effort. 


\section{References}

Bichsel, H., and E. D. Uehling, Multiple scattering correction for proton ranges and the evaluation of the L-shell correction and I value for Aluminum, Phys. Rev., 119(5), 1670-1680, 1960.

Eyges, L., Multiple scattering with energy loss, Phys. Rev., 74, 1534-1535, 1948.

Hogstrom, K. R., M. D. Mills, and R. Almond, Electron beam dose calculations, Phys. Med. Biol., 26(3), 445-459, 1981.

Hollmark, M., J. Uhrdin, Dz Belkie, I. Gudowska, and A. Brahme, Influence of multiple scattering and energy loss straggling on the absorbed dose distribution of therapeutic light ion beams: I. Analytical pencil model, Phys. Med. Biol., 49, 3247-3265, 2004.

Noshad, H., and N. Givechi, Proton therapy using the Monte Carlo method, Radiat. Meas., 39, 521-524, 2005.

Rossi, B., and K. Greisen, Cosmic-Ray Theory, Rev. Mod. Phys., 13, 240-315, 1941.

Scott, W. T., Correlated probabilities in multiple scattering, Phys. Rev., 76(2), 212-219, 1949.

Tweed, J., S. A. Walker, J. W. Wilson, F. A. Cucinotta, R. K. Tripathi, S. Blattnig, and C. J. Mertens, Computation methods for the HZETRN code, Adv Space Res., 25, 194201, 2005.

Wilson, J. W., et al., Transport Methods and Interactions for Space Radiations, NASA RP-1257, 1991.

Wilson, J. W., and H. Tai, Range and energy straggling in ion beam transport, NASA/TP2000-209864, 2000.

Wilson, J. W., J. Tweed, H. Tai, and R. K. Tripathi, A simple model for straggling evaluation, Nucl. Instrum. Methods B, 194, 389-392, 2002.

Wilson, J. W., R. K. Tripathi, C. J. Mertens, S. R. Blattnig, M. S. Clowdsley, F. A. Cucinotta, J. Tweed, J. H. Heinbockel, S. A. Walker, and J. E. Nealy, Verification and Validation: High Charge and Energy (HZE) Transport Codes and Future Development, NASA/TP-2005-000000, 2005. 


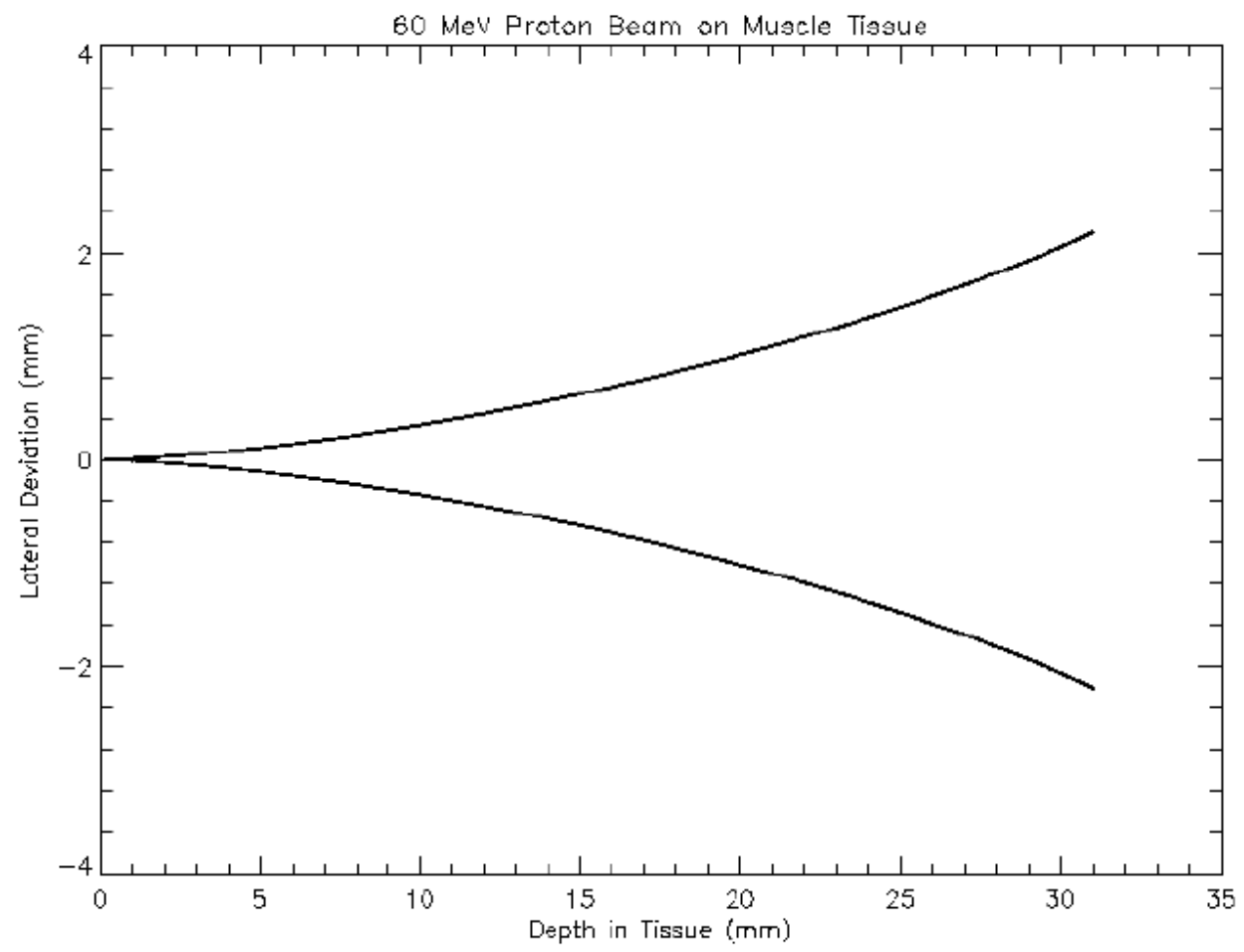

Figure 1: Radial broadening of an initial infinitesimal $60 \mathrm{MeV}$ proton beam on muscle tissue. The radial broadening is calculated out to $31 \mathrm{~mm}$ in tissue depth. The Bragg peak is located at $30.49 \mathrm{~mm}$ (see Figure 8). 


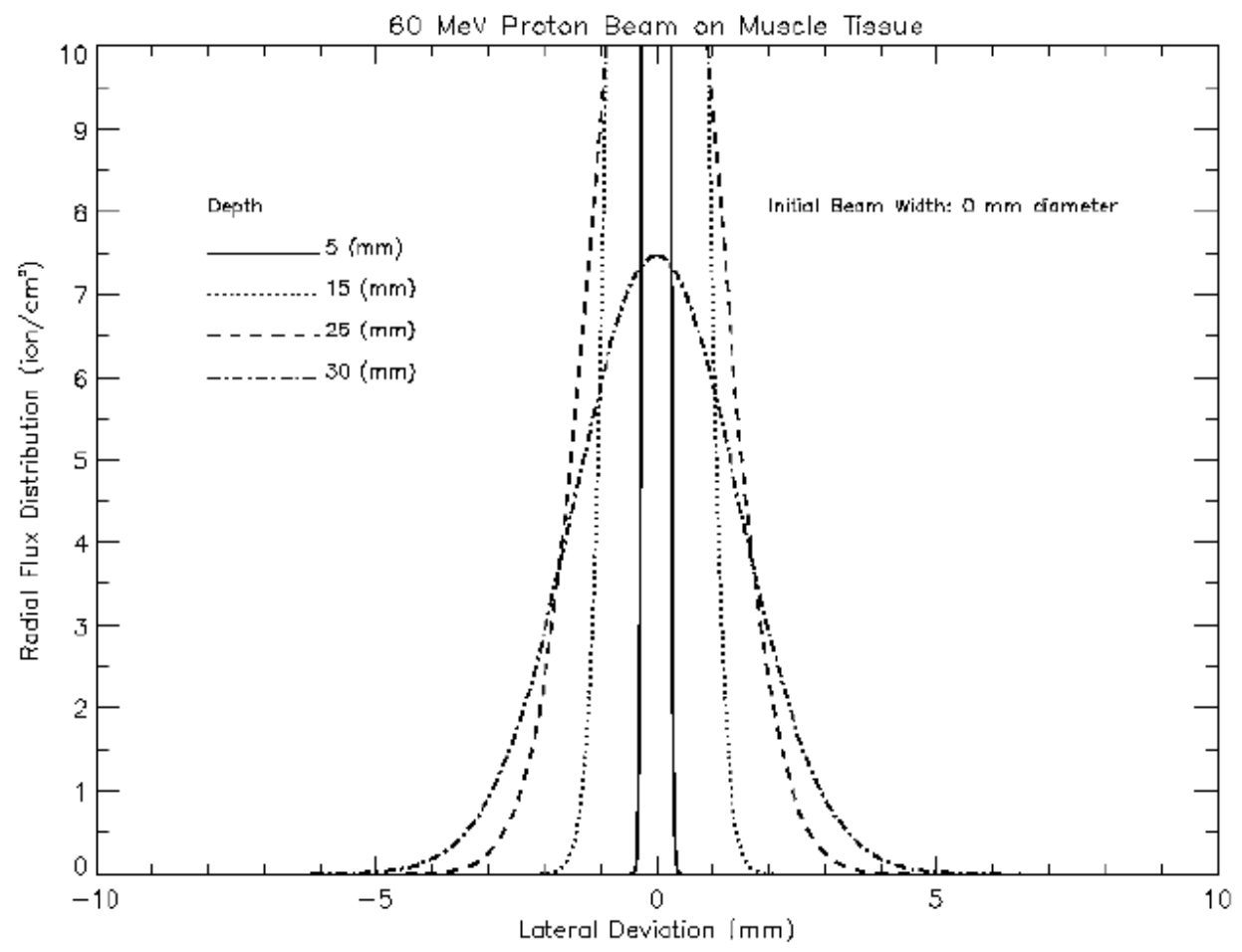

Figure 2: Radial flux distribution for an initial infinitesimal $60 \mathrm{MeV}$ proton beam on muscle tissue. The radial flux is shown along one of the lateral axis (i.e., either $x$ - or $y$-axis). The radial flux is shown at various depths in the muscle tissue. 


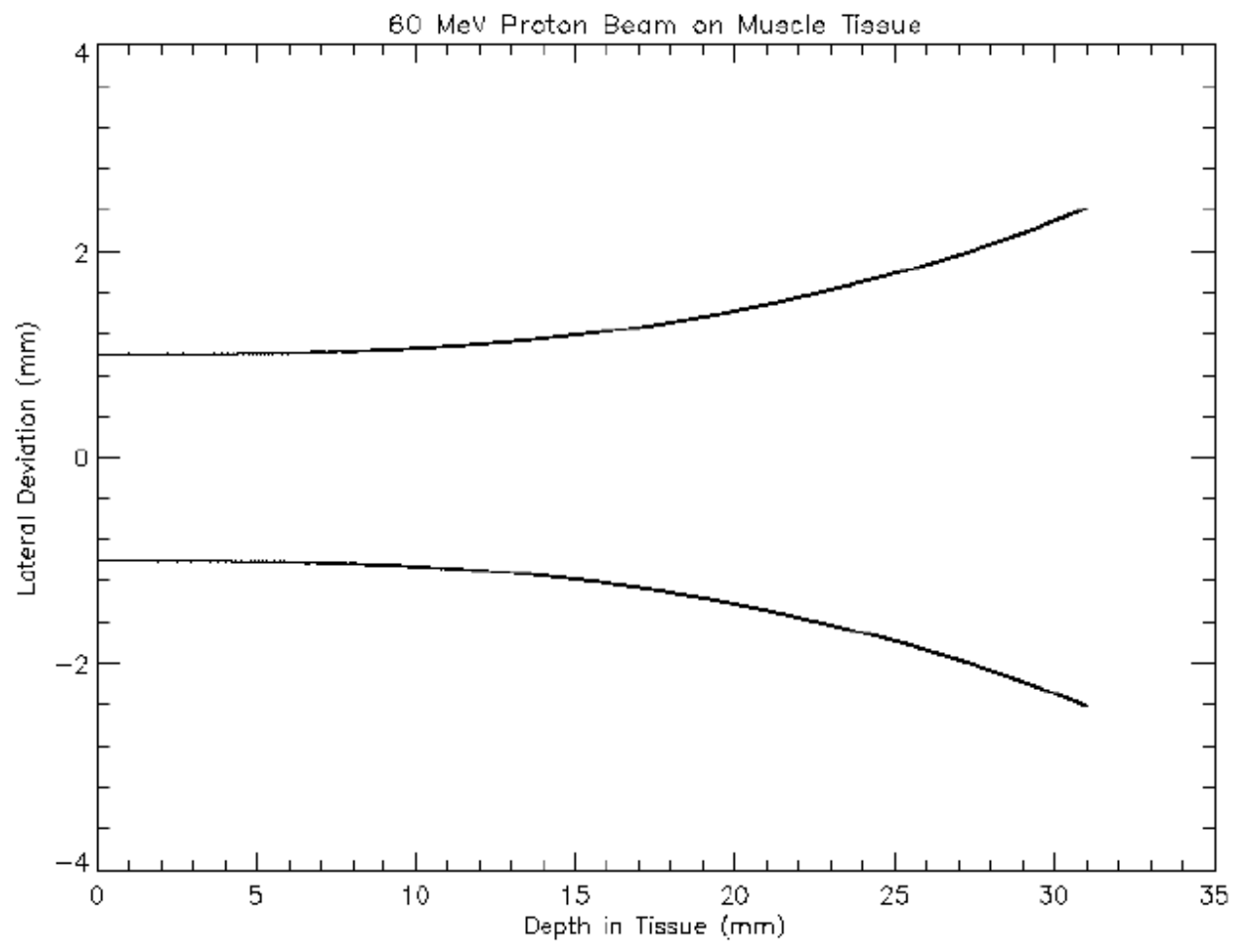

Figure 3: Radial broadening of a $60 \mathrm{MeV}$ proton beam on muscle tissue with an initial beam diameter of $2 \mathrm{~mm}$. The radial broadening is calculated out to $31 \mathrm{~mm}$ in tissue depth. The Bragg peak is located at $30.49 \mathrm{~mm}$ (see Figure 8). 


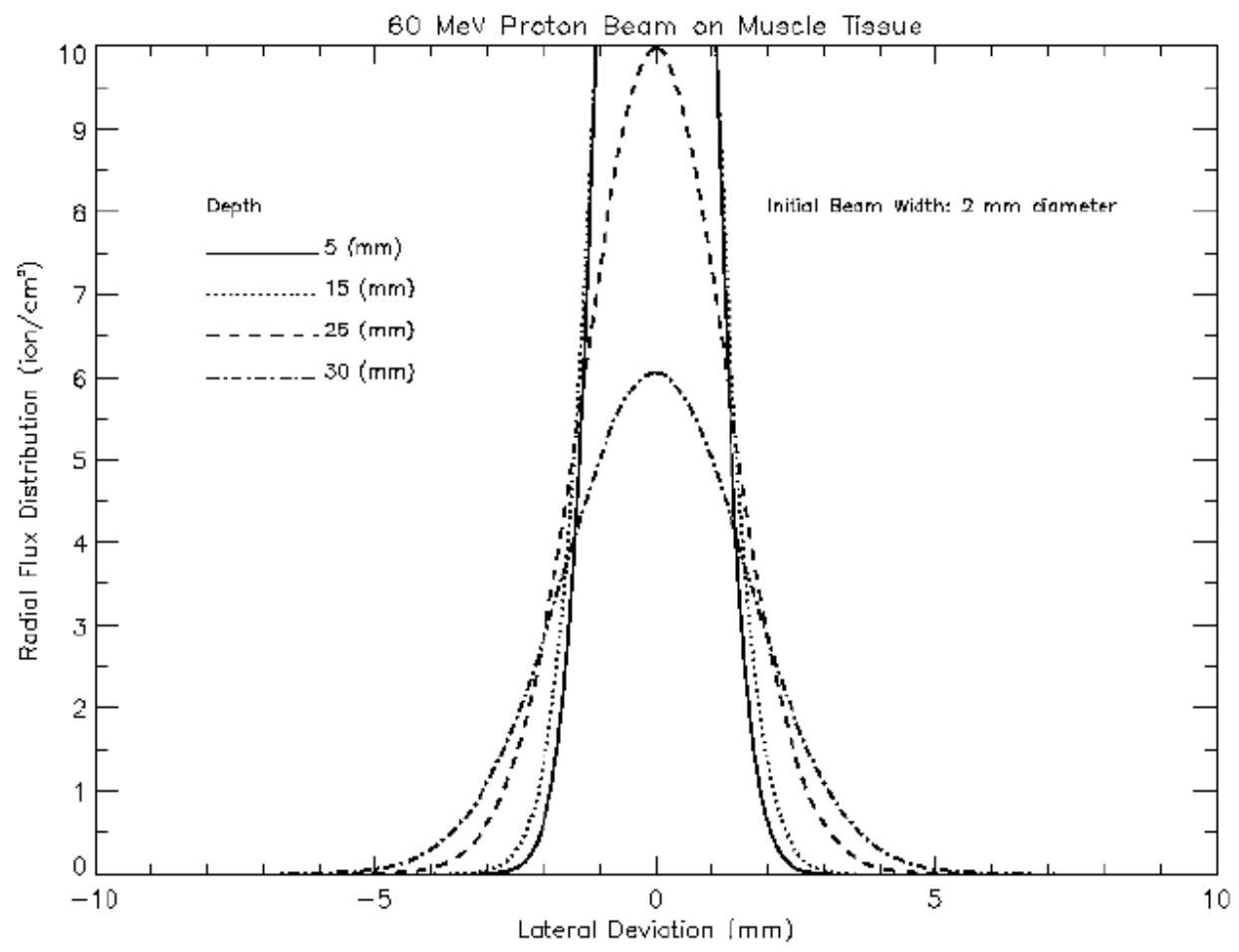

Figure 4: Radial flux distribution for a $60 \mathrm{MeV}$ proton beam on muscle tissue. The initial beam diameter is $\mathbf{2} \mathbf{~ m m}$. The radial flux is shown along one of the lateral axis (i.e., either the $x$ - or $y$-axis). The radial flux distribution is shown at various depths in the muscle tissue. 


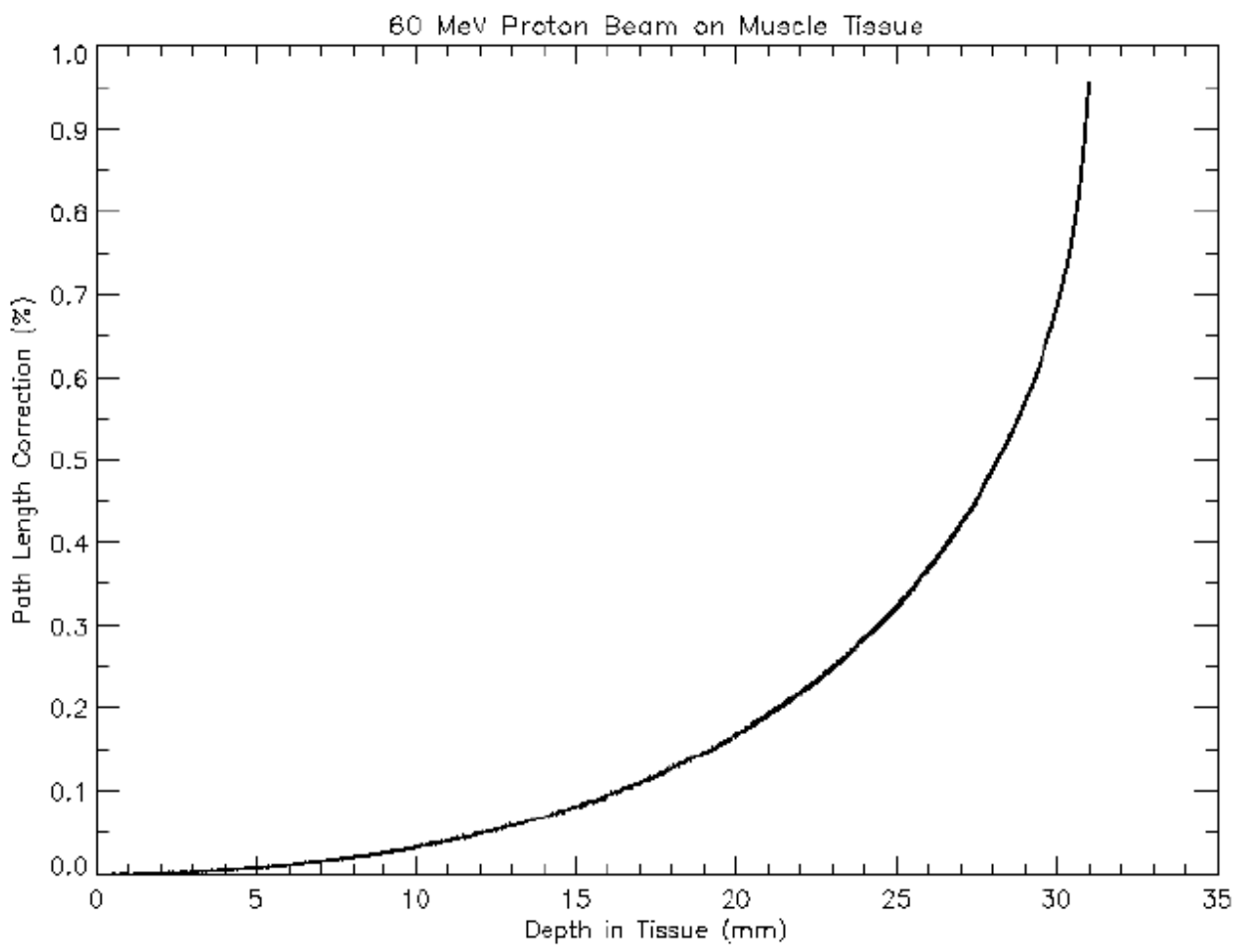

Figure 5: Percent increase in the effective 1-D path-length due to multiple scattering for a $60 \mathrm{MeV}$ proton beam on muscle tissue. Exiting ion beam assumed to have zero angular displacement, irrespective of lateral displacement. The percent increase in the effective 1-D path-length is calculated out to $31 \mathrm{~mm}$. The Bragg peak is located at $30.49 \mathrm{~mm}$ (see Figure 8). 


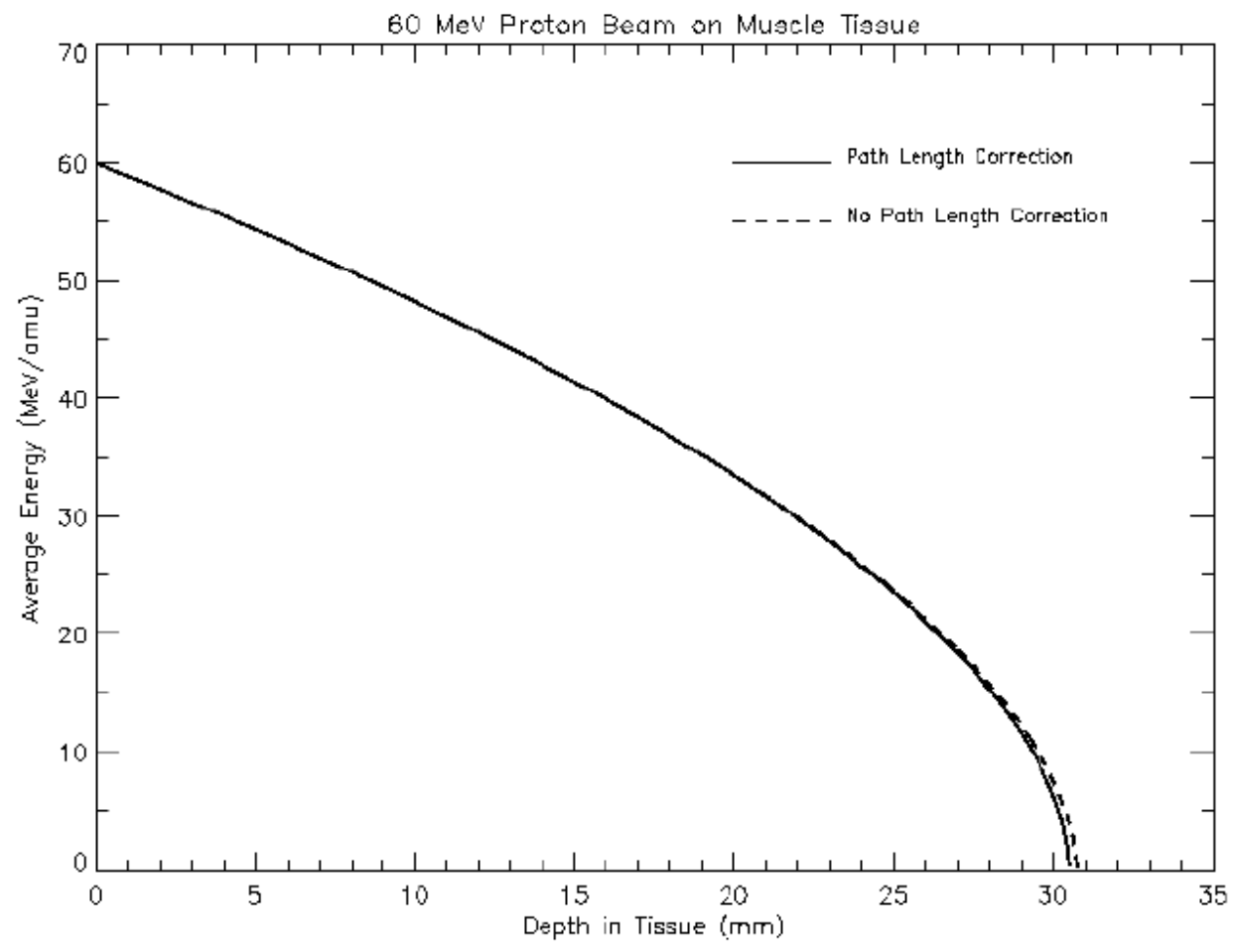

Figure 6: Mean energy as a function of depth for a $60 \mathrm{MeV}$ proton beam on muscle tissue. The mean energy is shown with and without the multiple scattering path-length correction applied. The Bragg peak is located at $30.49 \mathrm{~mm}$ when scattering effects are included. The Bragg peak is located at 30.73 $\mathrm{mm}$ when scattering is not included. 


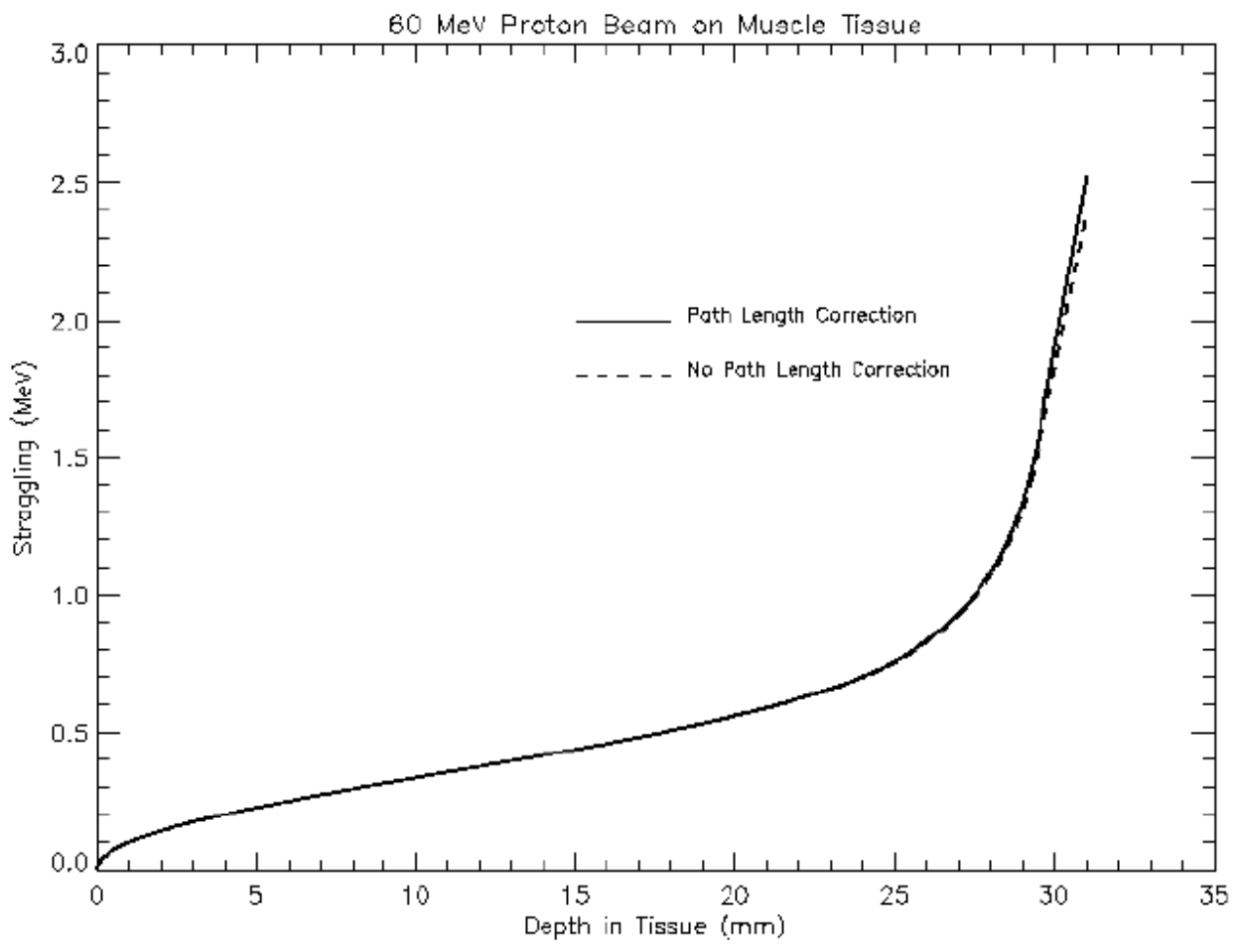

Figure 7: Energy straggling width as a function of depth for a $60 \mathrm{MeV}$ proton beam on muscle tissue. Straggling is shown with and without the multiple scattering path-length correction applied. The straggling width is calculated out to $31 \mathrm{~mm}$. The Bragg peak is located at $30.49 \mathrm{~mm}$ (see Figure 8). 


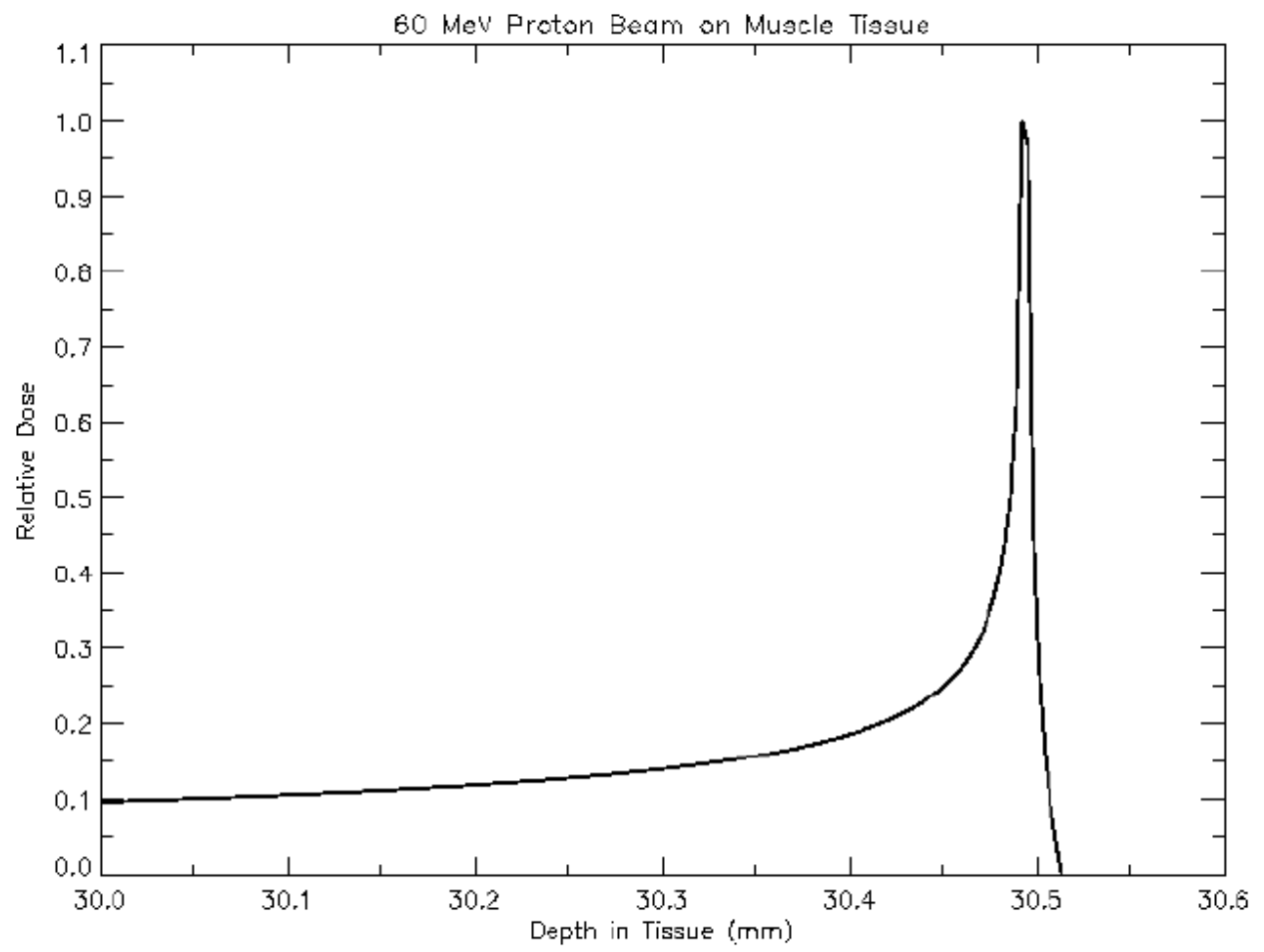

Figure 8: On-axis, relative dose-depth curve for a $60 \mathrm{MeV}$ proton beam on muscle tissue. Pathlength corrections due to multiple scattering are included (see (47)-(48) and the subsequent discussion). 


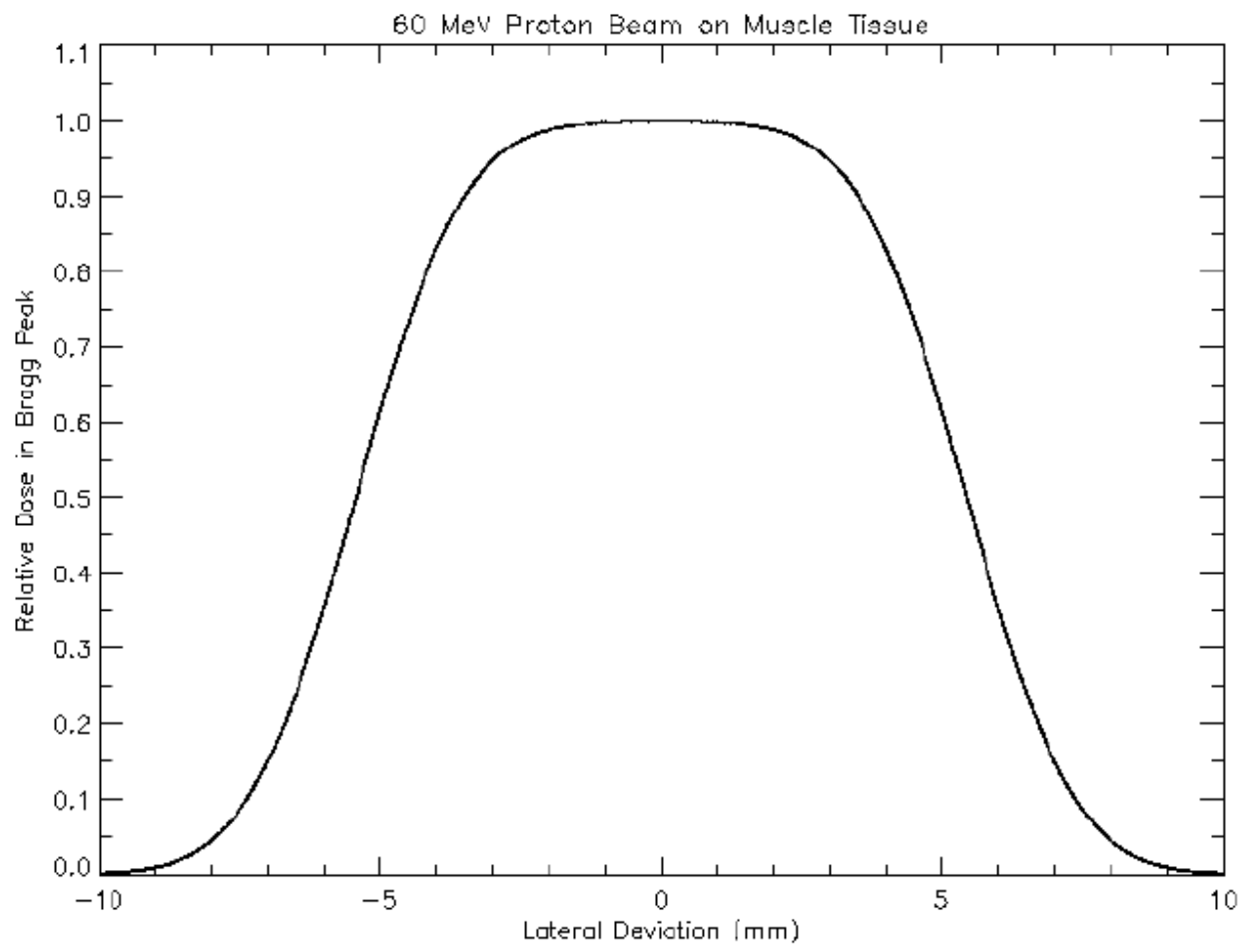

Figure 9: Lateral (relative) absorbed dose distribution at the Bragg peak (30.49 $\mathrm{mm}$ tissue depth) for a $60 \mathrm{MeV}$ proton beam on muscle tissue. The initial beam width is $10 \mathrm{~mm}$. 


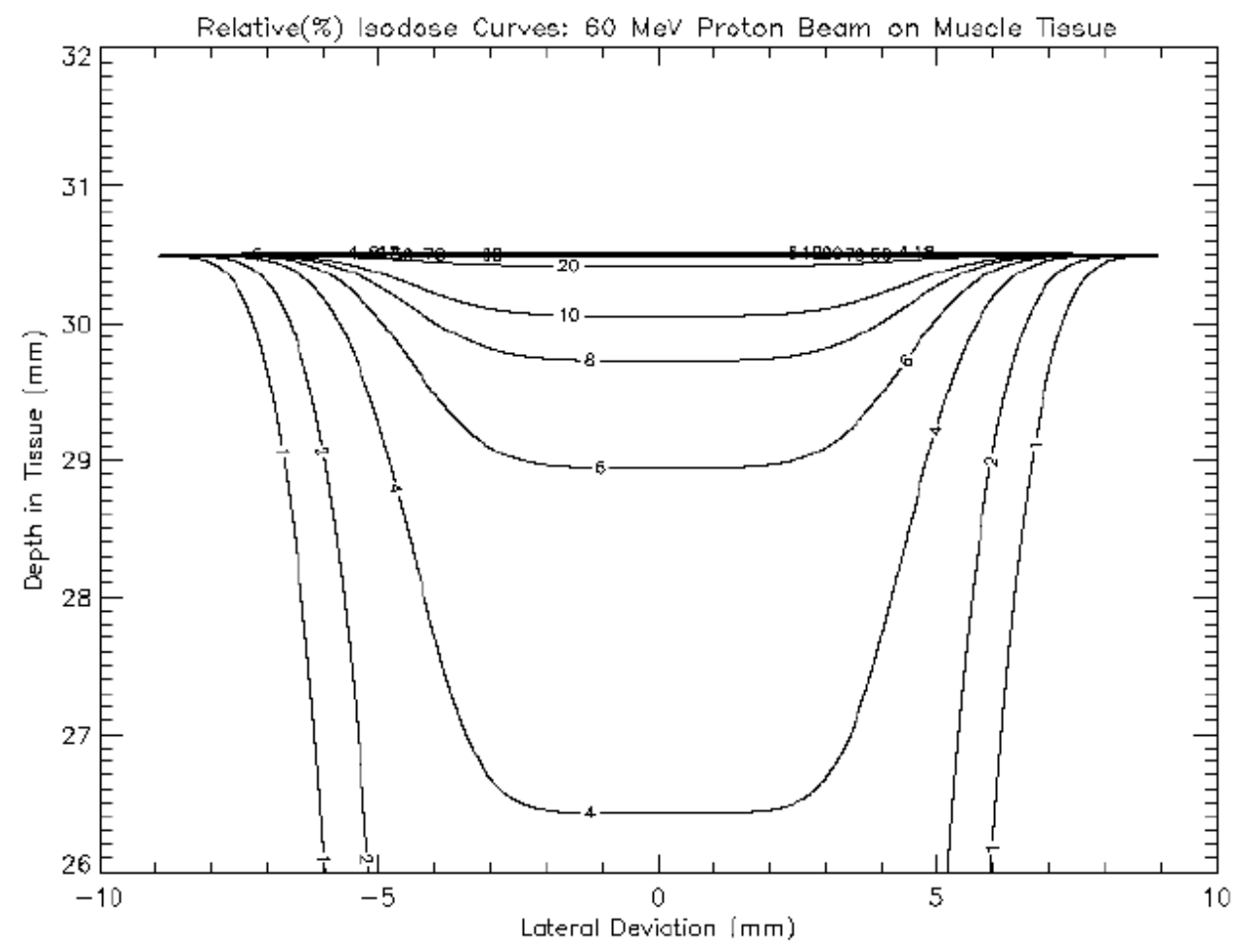

Figure 10: Relative isodose curves for a $60 \mathrm{MeV}$ proton beam on muscle tissue. The initial beam width is $10 \mathrm{~mm}$. 